\title{
The research of green residential investment project decision making
}

\author{
Hao Cheng ${ }^{1}$, Lei $\mathrm{Yu}^{2}$, ZhiMing $\mathrm{Hu}^{2}$ \\ ${ }^{1}$ School of Qingdao University of Technology, Qingdao 266520, China. \\ ${ }^{2}$ School of Qingdao University of Technology, Qingdao 266520,China. \\ E-mail: 2545802789@qq.com.
}

\begin{abstract}
People pay more attention to the green house recently, but the study of green building investment project decision-making is not enough. Considering the interaction between the attributes, Shapley function is used to determine the attribute weights, the integrated value is calculated using IG-IVIFHSA operator. Finally, through an actual case analysis to verify the feasibility and effectiveness of the given method .
\end{abstract}

\section{INTRODUCTION}

The idea of green architecture first appeared in early last century, and its core idea can be summarized as follows: in the process of architectural design and construction, it reflects the environment concern and sustainable development. In recent years, the concept of green building attracted widespread concern and attention, countries have established a green building evaluation system. China has also issued a corresponding green building evaluation system, but only limited to the building have been built to assess, it can't fundamentally solve the problem of high consumption of buildings.

Green buildings are widely concerned, most of the domestic scholars study green building evaluation. Such as Zang $^{[1]}$ designed a green building ecological index system; Qian ${ }^{[2]}$ improved the evaluation method of green building, and then determined the comprehensive evaluation level of green building. However, the researches mainly focused on value engineering ${ }^{[3]}$ and fuzzy comprehensive evaluation ${ }^{[4]}$.

\section{PRELIMINARIES}

At present, most of the research on multiple attribute decision making theory based on the importance of attributes is separated from each other, on the basis of its essentially corresponds to an additive measure. But it only gives the weights of evaluation indexes themselves, without considering the interaction between each other. Many scholars point out that in real life, the attribute importance between independent assumption is not set up. In fact, Sugeno fuzzy measure can deal with this problem.

\subsection{IG-IVIFHSA operator}

Definition: An IG-IVIFHSA operator of dimension $\mathrm{n}$ is a mapping

IG-IVIFHSA: $\Omega^{n} \rightarrow \Omega$ defined on the set of second arguments of two tuples $<u_{1}, \tilde{\alpha}_{1}>,<u_{2}, \tilde{\alpha}_{2}>, \ldots,<u_{n}, \tilde{\alpha}_{n}>$ with a set of order-inducing variables $u_{i}(i=1,2, \ldots, n)$ and a parameter $\gamma$ such that $\gamma \in(0,+\infty)$,denoted by

$\operatorname{IG-IVIFHSA~}_{\mu, v}\left(<u_{1}, \tilde{\alpha}_{1}>,<u_{2}, \tilde{\alpha}_{2}>, \ldots,<u_{n}, \tilde{\alpha}_{n}>\right)=\left(\frac{\bigoplus_{j=1}^{n} \varphi_{j}(\mu, N) \varphi_{\tilde{a}_{j}}^{\gamma}(v, A) \tilde{\alpha}_{j}^{\gamma}}{\sum_{j=1}^{n} \varphi_{j}(\mu, N) \varphi_{\tilde{a}_{j}}^{\gamma}(v, A)}\right)^{1 / \gamma}$

where $u_{j}$ is the $j$ th largest of $u_{i}(i=1,2, \ldots, n), \varphi_{j}(\mu, N)$ is the Shapley value with respect to (w.r.t.) the associated fuzzy measure $\mu$ on $N=\{1,2, \ldots, n\}$ for the $j$ th index, and $\varphi_{a_{j}}(v, A)$ is the Shapley value w.r.t. the fuzzy measure $v$ on $A=\left\{\tilde{\alpha}_{j}\right\}_{j=1, \ldots, n}$ for $\tilde{\alpha}_{j}(j=1,2, \ldots, n)$ 


\section{Green building investment decision-making index system}

We graded the Green ecological residential district construction key points and technical guidelines, found that the guideline for the green house evaluation is mainly based on reduce building energy consumption, resource conservation and environmental protection material. At the same time, with considering the economic benefits of projects investment and construction of the late operation and maintenance, the article establishes green residential investment decision-making system as shown in table 1 below.

TABLE 1 Index system diagram

\begin{tabular}{lcc}
\hline Policy makers & First grade indexes & The secondary indicators \\
\hline & Economic benefits & The cost of inputs \\
& Return on investment \\
Green residential & The payback period of investment \\
technology scheme & To reduce building energy consumption & New energy use \\
of the green house & Energy saving lighting & Energy efficiency \\
& Resources and materials & The recycling of water \\
& & Use environmental protection material \\
& Garbage recycling \\
& Intelligent system \\
& Eperation and maintenance & Equipment update rate \\
\hline
\end{tabular}

\section{Method based on the IG-IVIFHSA operator}

\subsection{The model of evaluation}

This section proposes a new approach to deal with interval-valued intuitionistic fuzzy multi-attribute decision making. It not only takes into account the importance of attributes and their ordered positions, but also reflects the correlation between attributes. When the weights of attributes and their ordered positions are given, we can apply the introduced aggregation operator to develop a method that can solve decision making. Firstly, we need to obtain the weights of them.

Let $\tilde{R}^{+}=\left(\tilde{r}_{1}^{+}, \tilde{r}_{2}^{+}, \ldots, \tilde{r}_{n}^{+}\right)$and $\tilde{R}^{-}=\left(\tilde{r}_{1}^{-}, \tilde{r}_{2}^{-}, \ldots, \tilde{r}_{n}^{-}\right)$respectively denote the positive and negative ideal vectors, where

$$
\begin{aligned}
& \tilde{r}_{j}^{+}=\left(\left[\max _{i} e_{i j}, \max _{i} f_{i j}\right],\left[\min _{i} g_{i j}, \min _{i} h_{i j}\right]\right) \\
& \tilde{r}_{j}^{-}=\left(\left[\min _{i} e_{i j}, \min _{i} f_{i j}\right],\left[\max _{i} g_{i j}, \max _{i} h_{i j}\right]\right)
\end{aligned}
$$

Let $d_{i j}=\frac{d_{i j}^{+}}{d_{i j}^{+}+d_{i j}^{-}}$, where

$$
\begin{aligned}
& d_{i j}^{+}=\frac{\left|e_{i j}-\max _{1 \leq i \leq m} e_{i j}\right|+\left|f_{i j}-\max _{1 \leq i \leq m} f_{i j}\right|+\left|g_{i j}-\min _{1 \leq i \leq m} g_{i j}\right|+\left|h_{i j}-\min _{1 \leq i \leq m} h_{i j}\right|}{4}, \\
& d_{i j}^{-}=\frac{\left|e_{i j}-\min _{1 \leq i \leq m} e_{i j}\right|+\left|f_{i j}-\min _{1 \leq i \leq m} f_{i j}\right|+\left|g_{i j}-\max _{1 \leq i \leq m} g_{i j}\right|+\left|h_{i j}-\max _{1 \leq i \leq m} h_{i j}\right|}{4} .
\end{aligned}
$$

Next, we consider the model for the optimal fuzzy measure on ordered set $\mathrm{N}$. For each $j=1,2, \ldots, n$, calculate 
$d\left(\tilde{r}_{i j}, \tilde{z}_{j}\right)=\left|e_{i j}-\sum_{i=1}^{m} e_{i j} / m\right|+\left|f_{i j}-\sum_{i=1}^{m} f_{i j} / m\right|+\left|g_{i j}-\sum_{i=1}^{m} g_{i j} / m\right|+\left|h_{i j}-\sum_{i=1}^{m} h_{i j} / m\right|$

for all $i=1,2, \ldots, \mathrm{m}$, where

$$
\tilde{z}_{j}=\left(\left[\frac{\sum_{i=1}^{m} e_{i j}}{m}, \frac{\sum_{i=1}^{m} f_{i j}}{m}\right],\left[\frac{\sum_{i=1}^{m} g_{i j}}{m}, \frac{\sum_{i=1}^{m} h_{i j}}{m}\right]\right) .
$$

Reorder $d\left(\tilde{r}_{i j}, \tilde{z}_{j}\right)$ in ascending order for each $i=1,2, \ldots, m$, we get

$$
d\left(\tilde{r}_{i(1)}, \tilde{z}_{i}\right) \leq d\left(\tilde{r}_{i(2)}, \tilde{z}_{i}\right) \leq \ldots \leq d\left(\tilde{r}_{i(n)}, \tilde{z}_{i}\right)
$$

where (.) is a permutation on $N$.

Based on the mean deviation method, we build the following model for the optimal fuzzy measure on ordered set $N$.

$$
\begin{gathered}
\max \sum_{i=1}^{m} \sum_{j=1}^{n} d\left(\tilde{r}_{i(j)}, \tilde{z}_{i}\right) \varphi_{j}(\mu, N), \\
\text { s.t. }\left\{\begin{array}{l}
\mu(N)=1 \\
\mu(S) \leq \mu(T) \quad \forall S, T \subseteq N \\
\mu(j) \in U_{j}, \mu(j) \geq 0 \forall j \in N
\end{array} .\right.
\end{gathered}
$$

where $\mu$ is the fuzzy measure on ordered set $N$, and $U_{j}$ is the range of the $j$ th position.

\subsection{The main decision procedure}

Step 1: If all attributes $c_{j}(j=1,2, \ldots, n)$ are benefit (i.e., the larger,the greater preference), then the attribute values do not need normalization. Otherwise, we normalize the IVIFV matrix

$\tilde{A}=\left(\tilde{\alpha}_{i j}\right)_{m \times n}$ into $\tilde{R}=\left(\tilde{r}_{i j}\right)_{m \times n}$

where $\tilde{r}_{i j}=\left\{\begin{array}{l}\tilde{a}_{i j} \text { for benefit attribute } C_{j} \\ \left(\tilde{a}_{i j}\right)^{C} \text { for cost attribute } C_{j}\end{array}\right.$, with $\tilde{\alpha}_{i j}^{C}=\left(\left[c_{i j}, d_{i j}\right],\left[a_{i j}, b_{i j}\right]\right)(i=1,2, \ldots, m ; j=1,2, \ldots, n)$.

Step 2: Solve the optimal fuzzy measure on attribute set $C$, and calculate the Shapley values of attri-butes .

Step 3: Solve the optimal fuzzy measure on ordered set $N$, and calculate the Shapley values of their ordered positions.

Step 4: Apply the induced generalized interval-valued intuitionis-tic fuzzy hybrid Shapley averaging (IG-IVIFHSA) operator

$\tilde{r}_{i}=$ IG-IVIFHSA $_{\mu, v}\left(<u_{1}, \tilde{r}_{i 1}>,<u_{2}, \tilde{r}_{i 2}>, \ldots,<u_{n}, \tilde{r}_{i n}>\right)=\left(\frac{\bigoplus_{j=1}^{n} \varphi_{j}(\mu, N) \varphi_{c_{j}}{ }^{\gamma}(v, C) \tilde{r}_{i j}{ }^{\gamma}}{\sum_{j=1}^{n} \varphi_{j}(\mu, N) \varphi_{c_{j}}{ }^{\gamma}(v, C)}\right)^{1 / \gamma}$ to get the comprehensive attribute value $\tilde{r}_{i}=\left(\left[u_{i}, v_{i}\right],\left[x_{i}, y_{i}\right]\right)(i=1,2, \ldots, m)$ of the alternative $a_{i}(i=1,2, \ldots, m)$, where $u_{j}$ is the $j$ th largest of 
$\mu_{i}(i=1,2, \ldots, \mathrm{m})$

Step 5: According to the comprehensive attribute value $\tilde{r}_{i}=\left(\left[u_{i}, v_{i}\right],\left[x_{i}, y_{i}\right]\right) \quad(i=1,2, \ldots, m)$,calculate the score $S\left(\tilde{r}_{i}\right)=\frac{u_{i}+v_{i}-x_{i}-y_{i}}{2}$ and the accuracy degree $H\left(\tilde{r}_{i}\right)=\frac{u_{i}+v_{i}+x_{i}+y_{i}}{2}$ to rank the alternative $a_{i}(i=1,2, \ldots, m)$, then to select the best one.

Step 6: End.

\section{Practical examples analysis}

Through data collection and analysis, and utilized solutions that obtained by IG - IVIFHSA operator comprehensive attribute value as follows, this paper calculated the value of comprehensive evaluation:

$F_{A}=[0.49,0.57][0.23,0.38], F_{B}=[0.42,0.52][0.22,0.38], F_{C}=[0.28,0.35][0.45,0.6]$.

$$
\begin{gathered}
S\left(F_{A}\right)=0.225, S\left(F_{B}\right)=0.17, S\left(F_{C}\right)=-0.21 . \\
F_{A}>F_{B}>F_{C} .
\end{gathered}
$$

Therefore, plan A is the best choice.

Different from traditional investment decision-making method, Lina Wang, who studies the sale of green residential investment decision from the perspective of value engineering. She used her method to solve this case and calculated that the ranking result is consistent with this article.

At the same time, application of traditional decision method of fuzzy comprehensive evaluation method and AHP, OWA operator to calculate the ranking results can see table 2:

TABLE 2 Different ways to sort results

\begin{tabular}{cl}
\hline Methods & Sorting result \\
\hline OWA operator & $F_{C}>F_{A}>F_{B}$ \\
AHP fuzzy comprehensive evaluation method & $F_{C}>F_{A}>F_{B}$ \\
Value engineering evaluation & $F_{A}>F_{B}>F_{C}$ \\
IG - IVIFHSA operator & $F_{A}>F_{B}>F_{C}$ \\
\hline
\end{tabular}

The table 2 shows that application of different methods to get the best solution may be different, policymakers can based on the actual needs to select the correct and ideal solutions.

\section{CONCLUSION}

The empirical research shows that the method of this paper can solve the problem of fuzzy information processing, the interaction between the index attributes and the weight setting in the incomplete information. Most of the current research on the assessment of green residential buildings are only based on green grade evaluation for completed buildings, and ignores the green residential construction project investment stage for different green technologies, this paper proposes an evaluation index system and the proposal of the corresponding decision method. The interval valued intuitionistic fuzzy Shapley function, and the corresponding optimization model is established to solve the problem, combined with the actual case gives the calculation method and steps, scheme comparison of ranking results finally and different decision-making methods. The results show that the optimal scheme can be different from that in the process of decision making, such as the interaction between the attributes and the importance of the attribute location.

\section{REFERENCES}

1. Peng Zang, Study on evaluation method of green building form the perspective of ecology, J. The BuildingScience. 30(2014) 19-23.

2. QinZhen Qian, Application of muli-level extension comprehensive method in green building evaluation, J. Journal of Engineering Management.28(2014) 11-16. 
3. YuHua An, Research on green building investment decision based on value engineering and fuzzy mathematics theory, J. Journal of Jilin University of Architecture.3(2015) 85-88.

4. Li-Na Wang, Research on green building investment decision based on Value Engineering(2010). 